\title{
A New Modified MR Dual Precision Positioning of Thin-Slice Oblique Sagittal Fat Suppression Proton Density Weighted Imaging: Its Diagnostic Accuracy in Anterior Cruciate Ligament Injury and its Grades
}

Kun Li

First Affiliated Hospital of Soochow University

Felix Young Jhonatan

First Affiliated Hospital of Soochow University

Zhaohui Yu

First Affiliated Hospital of Soochow University

Jinhua Chen

First Affiliated Hospital of Soochow University

Lixin Huang

First Affiliated Hospital of Soochow University

Huilin Yang

First Affiliated Hospital of Soochow University

Jun Du ( $\nabla$ dujun5166@163.com)

First Affiliated Hospital of Soochow University

\section{Research Article}

Keywords: Anterior cruciate ligament,Dual precision positioning of thin-slice oblique sagittal fat suppression proton density-weighted imaging,Magnetic resonance imaging,Diagnostic accuracy,Arthroscopy

Posted Date: December 15th, 2021

DOI: https://doi.org/10.21203/rs.3.rs-1119448/v1

License: (9) (i) This work is licensed under a Creative Commons Attribution 4.0 International License. Read Full License 


\section{Abstract}

Purpose To evaluate the diagnostic accuracy of a new modified MR dual precision positioning of thinslice oblique sagittal fat suppression proton density-weighted imaging (DPP-TSO-Sag-FS-PDWI) sequence in detecting ACL injuries and its grades compared to standard sequences using arthroscopy as the standard reference.

Materials and Methods 42 patients enrolled in this retrospective study received the 1.5-T MRI with standard sequences and the new modified DPP-TSO-Sag-FS-PDWI sequence, and their arthroscopy results was recorded. The Mc Nemer-Bowker and weighted Kappa was performed to compare the consistency of MRI diagnosis with arthroscopic results. Finally, the diagnostic accuracy was calculated based on the true positive, true negative, false negative and false positive values.

Results The diagnostic consistency of the DPP-TSO-Sag-FS-PDWI were higher than standard sequences for both reader 1 ( $K=0.876$ vs. 0.620$)$ and reader $2(K=0.833$ vs. 0.683$)$ with good diagnostic repeatability ( $K=0.794$ vs. 0.598 ). Furthermore, the DPP-TSO-Sag-FS-PDWI can classify and diagnose three grades of ACL injury [the sensitivity, specificity, accuracy, positive predictive value (PPV) and negative predictive value were more than $84 \%]$, especially for grade II injury as the PPV was superior for reader 1 (92.3\% vs. $53.9 \%)$ and reader 2 (84.6\% vs. $69.2 \%)$.

Conclusion The new modified DPP-TSO-Sag-FS-PDWI sequence can display the ACL injury on one or continuous levels by maximizing the acquisition of complete ligament shape and true anatomical images, and excluding the influence of anatomical differences between individuals. It can improve the diagnostic accuracy with good repeatability and classify three grades of the ACL injury.

\section{Introduction}

Anterior cruciate ligament $(\mathrm{ACL})$ arises from the medial surface of the lateral femoral condyle and extends the oblique course to the medial and anterior portion in the central area of the tibial plateau [1]. The ligament is divided into two bundles, including the anteromedial bundle and the posterolateral bundle. The function of ACL is to maintain anterior tibial movement and prevent excessive tibial rotation [2]. The ACL injury is the most common ligament injury in the United States, with 100,000 to 200,000 yearly [3]. Internal or external rotation and valgus or varus movements increase the risk of ACL damage [3, 4]. Meanwhile, a majority of ACL injuries occur when the knee is fully stretched and sudden fall $[3,2]$. A complete tear of ACL mainly occurs in femoral insertion due to anterior tibial displacement and increased internal rotation, resulting in instability of the knee joint and a significant increase in rates of damage to menisci or articular cartilage [5,3].

The examination under anesthesia and visual investigation at the knee arthroscopy are the gold standard by observing the internal structure of the joint, but most patients undergo magnetic resonance imaging (MRI) before arthroscopic treatment [6]. MRI is an effective method for the diagnosis of knee injury because it's non-invasive and lacks of ionizing radiation to the patients $[7,8]$. It also minimized the 
opportunity for unnecessary arthroscopic surgery, which required high financial needs and possibly trauma [9]. MRI displayed the injured site with excellent soft-tissue contrast and high-resolution image with wide range parameter and direct observation of $\operatorname{ACL}$ morphologic characteristics $[10,8,9]$. However, the limitation of standard MRI sequences [including sagittal and coronal $T_{1}$-weighted imaging $\left(T_{1} \mathrm{WI}\right)$, $\mathrm{T}_{2} \mathrm{WI}$ and fat suppression proton density-weighted imaging (FS-PDWI)] is that it produces partial volume effect caused by relatively slice thickness (3-5mm) and increased gap (10-30\% of the thickness) between slices [11, 12], which is a difficult challenge to diagnose partial tear and the diagnostic accuracy was relatively low $[13,14]$.

A sagittal-oblique technique has been proposed to standard sequences for the detection of partial ACL tears. However, these sequences have not been assessed for diagnostic accuracy based on arthroscopy as a standard [15]. Therefore, we added a new modified dual precision positioning of thin-slice oblique sagittal fat suppression proton density-weighted imaging (DPP-TSO-Sag-FS-PDWI) to standard sequences to improve the diagnostic accuracy by enhancing the visualization of ACL natural anatomical structure (scanning along the ACL anatomical direction on both axial and coronal planes), and eliminating partial volume effects (reducing the slice thickness from $5 \mathrm{~mm}$ to $2.3 \mathrm{~mm}$ and thickness gap from $0.5 \mathrm{~mm}$ to $0 \mathrm{~mm}$ ). The purpose of this study was to evaluate the diagnostic accuracy of a new modified DPP-TSO-Sag-FS-PDWI sequence in detecting ACL injuries and its different grades compared to standard sequences using arthroscopy as the standard reference.

\section{Material And Methods}

\section{Study population}

This retrospective study was conducted according to the guidelines of the Declaration of Helsinki, and approved by the Institutional Ethics Committee of the First Affiliated Hospital of Soochow University (protocol code No.155, 13th of July 2020). Informed consent was waived by the Institutional Ethics Committee of our hospital due to the retrospective nature of the study and the use of anonymous clinical data. We searched the data of patients from March 1, 2018, to March 1, 2020, who received MRI examination of the knee joint and arthroscopy treatment. We excluded patients without the new modified DPP-TSO-Sag-FS-PDWI sequence, the scanning procedure is performed in other institutions and without arthroscopic treatment. Finally, forty-two eligible patients were included in the study, all of whom performed MRI examination with the new modified DPP-TSO-Sag-FS-PDWI sequence and recorded arthroscopic treatment during surgery (Table 1). 
Table 1

Clinical data of the participants with MRI examination and arthroscopy results

\begin{tabular}{|c|c|c|c|c|c|c|c|c|}
\hline Sex & Age & $\begin{array}{l}\text { Right } \\
\text { knee }\end{array}$ & $\begin{array}{l}\text { Left } \\
\text { knee }\end{array}$ & Grade of injury & 0 & I & II & III \\
\hline 33 Men & $29 \pm 7(19-54)$ & 20 & 13 & Arthroscopy & 2 & 3 & 13 & 24 \\
\hline \multirow[t]{4}{*}{$\begin{array}{l}9 \\
\text { Women }\end{array}$} & $\begin{array}{l}31 \pm 12(14- \\
52)\end{array}$ & 3 & 6 & Standard Sequence 1 & 3 & 4 & 8 & 27 \\
\hline & & & & Standard Sequence 2 & 3 & 5 & 12 & 22 \\
\hline & & & & $\begin{array}{l}\text { DPP-TSO-Sag-FS-PDWI } \\
1\end{array}$ & 2 & 3 & 14 & 23 \\
\hline & & & & $\begin{array}{l}\text { DPP-TSO-Sag-FS-PDWI } \\
2\end{array}$ & 2 & 3 & 13 & 24 \\
\hline $\begin{array}{l}\text { DPP-TSC } \\
\text { proton d }\end{array}$ & $\begin{array}{l}\text { ag-FS-PDWI, Du } \\
\text { ity weighted im }\end{array}$ & $\begin{array}{l}\text { precisi } \\
\text { ging; } 1,\end{array}$ & $\begin{array}{l}\text { itionin } \\
1 ; 2, \mathrm{~F}\end{array}$ & $\begin{array}{l}\text { in-slice oblique sagittal } f \\
2 \text {. }\end{array}$ & & & & \\
\hline
\end{tabular}

\section{Image acquisition}

MRI scanning was performed on a 1.5-T superconducting magnetic resonance scanner (Magnetom Espree, A Tim System; Siemens, Erlangen, Germany) with a single channel knee coil. The patients were scanned in the supine position and their legs were straightened. The knee joint was fixed by sandbags on both sides and the lower edge of the patella was placed in the center of the coil. The scanning range was initiated from the distal end of the femur to the proximal end of the tibia and fibula, including the entire knee joint. The conventional MR scanning sequences and scanning order are axial fat suppression proton density-weighted imaging (FS-PDWI) [repetition time/echo time (TR/TE), $2970 \mathrm{~ms} / 36 \mathrm{~ms}$; field of view (FOV), $180 \mathrm{~mm}$; matrix size, $256 \times 256$; slice thickness/slice spacing, $4.0 \mathrm{~mm} / 1.2 \mathrm{~mm}$, sagittal $\mathrm{T}_{1} \mathrm{WI}$ (TR/TE, $350 \mathrm{~ms} / 11 \mathrm{~ms}$; FOV, 160mm; matrix size, $320 \times 320$; slice thickness/slice spacing, $4.0 \mathrm{~mm} / 0.6$ $\mathrm{mm}$ ), sagittal FS PDWI (TR/TE, $2340 \mathrm{~ms} / 43 \mathrm{~ms}$; FOV, 160mm; matrix size, $256 \times 256$; slice thickness/slice spacing, $4.0 \mathrm{~mm} / 0.6 \mathrm{~mm})$, coronal $\mathrm{T}_{2} \mathrm{WI}(\mathrm{TR} / \mathrm{TE}, 3052 \mathrm{~ms} / 63 \mathrm{~ms}$; FOV, $160 \mathrm{~mm}$; matrix size, $320 \times 320$; slice thickness/slice spacing, $4.0 \mathrm{~mm} / 0.6 \mathrm{~mm}$ ) and coronal FS PDWI (TR/TE, $2340 \mathrm{~ms} / 43 \mathrm{~ms}$; FOV, $160 \mathrm{~mm}$; matrix size, $256 \times 256$; slice thickness/slice spacing, $4.0 \mathrm{~mm} / 0.6 \mathrm{~mm})$. Finally, the DPP-TSO-SagFS-PDWI sequence (TR/TE, $2000 \mathrm{~ms} / 42 \mathrm{~ms}$; FOV, 160mm; matrix size, $320 \times 320$; slice thickness/slice spacing, $2.3 \mathrm{~mm} / 0 \mathrm{~mm}$ ) was acquired along the direction of ACL with the axial FS-PDWI and coronal FSPDWI as the scanning position map, which took 3 minutes and 24 seconds. The scanning method and the performance of ACL were shown in Fig. 1.

\section{Image analysis}

All images were transmitted to Picture Archiving and Communication Systems (PACS) and independently reviewed by two musculoskeletal radiologists (the first reader with 8 years of experience and the second reader with 6 years of experience). They were blinded to clinical and arthroscopic results. The reading 
procedure started with standard MRI sequences and followed by combining the new modified DPP-TSOSag-FS-PDWI sequence into the standard sequences. The ACL injury on the MRI is divided into three grades according to the diagnostic criteria: grade I injury is a partial tear with less than half of ligament disrupted; grade II injury is a partial tear with more than half part of the ligament tear; grade III was a complete tear [16].

\section{Arthroscopy protocol}

The arthroscopy operation was performed by using American Smith \& Nephew arthroscopic device. The procedure was performed by a senior surgeon with more than 10 years of experience in sports medicine and aware of MRI results and clinical reports. The ACL tears were classified into three levels according to the American Academy of Orthopedic Surgeons (AAOS) grading system: grade I is a sprain, mildly damaged and slightly stretched, but it can still keep the knee joint stable; grade II is a sprain and the ligament is stretched or loose, which is also known as partial tear; grade III is a sprain with complete tear, that is, the ligament breaks into two parts and the knee joint is unstable (https://orthoinfo.aaos.org/en/diseases--conditions/anterior-cruciate-ligament-acl-injuries/).

\section{Statistical analysis}

The frequency of MRI diagnosis was counted, including standard MRI sequences and the new modified DPP-TSO-Sag-FS-PDWI sequences, compared to arthroscopy results. The $\chi^{2}$ test of paired comparison (Mc Nemer-Bowker) and weighted Kappa test was performed to compare the consistency of MRI diagnosis with arthroscopic results and the twice observations. When the kappa value $\leq 0.20$, it indicates slight consistency; 0.21 to 0.40 , fair consistency; 0.61 to 0.80 , substantial consistency; 0.81 to 0.99 , almost perfect consistency and 1.0, perfect consistency [17]. The sensitivity (SE), specificity (SP), accuracy (AC), positive predictive value (PPV), and negative predictive value (NPV) of MRI diagnosis were calculated for Grade I, Grade II, and Grade III based on true positive (TP), true negative (TN), false positive (FP), and false-negative (FN). The Statistic Package for Social Sciences (SPSS, version 25.0, Chicago, IL) software was used for statistical analysis, and $p$ values $<0.05$ were considered significant for 2-tailed probability.

\section{Results}

\section{Patients' characteristics}

A total of 42 patients were analyzed in our retrospective study. Clinical data of the patients with the results of twice MRI observations and arthroscopy examination were shown in Table 1.Forty patients suffered an ACL injury and two patients suffered meniscus injury with normal ACL (suspected ACL injury before arthroscopy). There were 33 men with an average age ( \pm standard deviation) of $29 \pm 7$ years and 9 women with $31 \pm 12$ years. According to the medical records, $73.8 \%$ (31/42) of them suffered from sports injuries and the rest $26.2 \%(21 / 42)$ patients suffered from accidents. 


\section{MRI and arthroscopy manifestations in different grade of ACL injury}

Standard MRI sequences and the new modified DPP-TSO-Sag-FS-PDWI sequences showed similar results for the two normal ACLs, and the appearance of ligaments had normal signal intensity without any separation. Complete ligament shape and its low signal intensity could be visualized on one or continuous layers of the new modified DPP-TSO-Sag-FS-PDWI sequence. The arthroscopic finding revealed the normal ligament was tight and tilted from the tibia to the condyle in the oblique course (Fig. 2).

Grade I injury of ACL is mild injury. The shape of the injured ligament was similar to the normal ligament on standard sequences. On the new modified DPP-TSO-Sag-FS-PDWI sequence, the ligament was tortuous with localized increased signal intensity. Arthroscopy showed the not torn ligament with local bleeding and its pulling force was reduced (Fig. 3).

The grade II injury of ACL showed partial tear with morphological change on standard sequences, it also manifested as local swelling, partial thinning of the ligament, and unclear tearing. Similar findings were also present on the new modified DPP-TSO-Sag-FS-PDWI sequence, but there was a separation in the part of the ligament. During the arthroscopic examination, grade II injury was characterized by partial tear with free edge and loose ligament stretch and tension (Fig. 4).

The grade III injury of ACL on standard sequences and the new modified DPP-TSO-Sag-FS-PDWI sequence showed the absence of ligament integrity. The ligament was completely torn into two parts and the separation was obvious. Arthroscopic examination revealed a complete tear of the ligament (Fig. 5).

\section{Comparison of diagnostic consistency between MRI results and arthroscopy}

We compared the diagnostic consistency of standard sequences and the new modified DPP-TSO-Sag-FSPDWI sequence with arthroscopy for the first reader and second reader (Table 2). There was a fair consistency between standard sequences and arthroscopy in both reader 1 ( $K=0.620$, from 0.449 to $0.855)$ and reader 2 ( $K=0.683$, from 0.436 to 0.844$)$. The consistency between the new modified DPPTSO-Sag-FS-PDWI sequence and arthroscopy were almost perfect (reader $1: K=0.876$, from 0.707 to 1 ; reader 2: $K=0.833$, from 0.696 to 0.996 ). 
Table 2

The diagnostic consistency comparison of standard MRI sequences and the new modified DPP-TSO-SAGFS-PDWI sequence with arthroscopy, and standard sequences with the new modified DPP-TSO-SAG-FS-

PDWl sequence for reader 1 and reader 2

\begin{tabular}{|c|c|c|c|c|c|c|c|c|c|}
\hline \multicolumn{2}{|c|}{$\begin{array}{l}\text { Diagnostic consistency } \\
\text { comparison }\end{array}$} & \multicolumn{2}{|c|}{$\begin{array}{l}\text { Standard } \\
\text { sequences } 1 \\
\text { and } \\
\text { Arthroscopy }\end{array}$} & \multicolumn{2}{|c|}{$\begin{array}{l}\text { Standard } \\
\text { sequences } 2 \\
\text { and } \\
\text { Arthroscopy }\end{array}$} & \multicolumn{2}{|c|}{$\begin{array}{l}\text { DPP-TSO-Sag- } \\
\text { FS-PDWI } 1 \text { and } \\
\text { Arthroscopy }\end{array}$} & \multicolumn{2}{|c|}{$\begin{array}{l}\text { DPP-TSO-Sag- } \\
\text { FS-PDWI } 2 \text { and } \\
\text { Arthroscopy }\end{array}$} \\
\hline \multirow{3}{*}{$\begin{array}{l}\text { Standard } \\
\text { sequences } \\
\text { and DPP- } \\
\text { TSO-Sag- } \\
\text { FS-PDWl } \\
\text { comparied } \\
\text { with } \\
\text { arthroscopy }\end{array}$} & $\begin{array}{l}\text { McNemar- } \\
\text { Bowker } \\
\text { test }\end{array}$ & $\begin{array}{l}\chi^{2}= \\
5.571\end{array}$ & $\begin{array}{l}p= \\
0.134\end{array}$ & $\begin{array}{l}\chi^{2}= \\
3.200\end{array}$ & $\begin{array}{l}p= \\
0.362\end{array}$ & $\begin{array}{l}\chi^{2}= \\
0.333\end{array}$ & $\begin{array}{l}p= \\
0.564\end{array}$ & $\begin{array}{l}x^{2}= \\
0.000\end{array}$ & $\begin{array}{l}p= \\
1.000\end{array}$ \\
\hline & $\begin{array}{l}\text { Weighted } \\
\text { Kappa }\end{array}$ & $\begin{array}{l}K= \\
0.620\end{array}$ & $\begin{array}{l}p= \\
0.000\end{array}$ & $\begin{array}{l}K= \\
0.683\end{array}$ & $\begin{array}{l}p= \\
0.000\end{array}$ & $\begin{array}{l}K= \\
0.876\end{array}$ & $\begin{array}{l}p= \\
0.000\end{array}$ & $\begin{array}{l}K= \\
0.833\end{array}$ & $\begin{array}{l}p= \\
0.000\end{array}$ \\
\hline & & $\begin{array}{l}(0.449 \\
\text { to } \\
0.855)\end{array}$ & & $\begin{array}{l}(0.436 \\
\text { to } \\
0.844)\end{array}$ & & $\begin{array}{l}(0.707 \\
\text { to } 1)\end{array}$ & & $\begin{array}{l}(0.696 \\
\text { to } \\
0.996)\end{array}$ & \\
\hline \multicolumn{2}{|c|}{$\begin{array}{l}\text { Diagnostic consistency } \\
\text { comparison }\end{array}$} & \multicolumn{2}{|c|}{$\begin{array}{l}\text { Standard } \\
\text { sequences } 1 \\
\text { and DPP-TSO- } \\
\text { Sag-FS-PDWI } 1\end{array}$} & \multicolumn{2}{|c|}{$\begin{array}{l}\text { Standard } \\
\text { sequences } 2 \\
\text { and DPP-TSO- } \\
\text { Sag-FS-PDWI } 2\end{array}$} & \multicolumn{2}{|c|}{$\begin{array}{l}\text { Standard } \\
\text { sequences } 1 \\
\text { and Standard } \\
\text { sequences } 2\end{array}$} & \multicolumn{2}{|c|}{$\begin{array}{l}\text { DPP-TSO-Sag- } \\
\text { FS-PDWI } 1 \text { and } \\
\text { DPP-TSO-Sag- } \\
\text { FS-PDWI } 2\end{array}$} \\
\hline \multirow{3}{*}{$\begin{array}{l}\text { Standard } \\
\text { sequences } \\
\text { comparied } \\
\text { with DPP- } \\
\text { TSO-Sag- } \\
\text { FS-PDWl } \\
\text { sequence }\end{array}$} & $\begin{array}{l}\text { McNemar- } \\
\text { Bowker } \\
\text { test }\end{array}$ & $\begin{array}{l}\chi^{2}= \\
7.000\end{array}$ & $\begin{array}{l}p= \\
0.072\end{array}$ & $\begin{array}{l}\chi^{2}= \\
4.000\end{array}$ & $\begin{array}{l}p= \\
0.261\end{array}$ & $\begin{array}{l}\chi^{2}= \\
4.571\end{array}$ & $\begin{array}{l}p= \\
0.206\end{array}$ & $\begin{array}{l}\chi^{2}= \\
0.200\end{array}$ & $\begin{array}{l}p= \\
0.655\end{array}$ \\
\hline & $\begin{array}{l}\text { Weighted } \\
\text { Kappa }\end{array}$ & $\begin{array}{l}K= \\
0.710\end{array}$ & $\begin{array}{l}p= \\
0.000\end{array}$ & $\begin{array}{l}K= \\
0.722\end{array}$ & $\begin{array}{l}p= \\
0.000\end{array}$ & $\begin{array}{l}K= \\
0.598\end{array}$ & $\begin{array}{l}p= \\
0.000\end{array}$ & $\begin{array}{l}K= \\
0.794\end{array}$ & $\begin{array}{l}p= \\
0.000\end{array}$ \\
\hline & & $\begin{array}{l}(0.544 \\
\text { to } \\
0.916)\end{array}$ & & $\begin{array}{l}(0.512 \\
\text { to } \\
0.880)\end{array}$ & & $\begin{array}{l}(0.338 \\
\text { to } \\
0.805)\end{array}$ & & $\begin{array}{l}(0.537 \\
\text { to } \\
0.923)\end{array}$ & \\
\hline
\end{tabular}

DPP-TSO-Sag-FS-PDWI, Dual precision positioning of thin-slice oblique sagittal fat suppression proton density weighted imaging; 1 , Reader $1 ; 2$, Reader 2 .

The diagnostic consistency comparison between standard sequences and the new modified DPP-TSOSag-FS-PDWI sequence for both readers were presented in Table 2. There was a fair consistency between them in both reader 1 ( $K=0.710$, from 0.544 to 0.916$)$ and reader $2(K=0.722$, from 0.512 to 0.880$)$. Nevertheless, the diagnostic consistency of standard sequences was only 0.598 (0.338 to 0.805$)$ and the new modified DPP-TSO-Sag-FS-PDWI sequence was 0.794 (0.537 to 0.923), thus indicating good diagnostic repeatability of the new modified DPP-TSO-Sag-FS-PDWI sequence.

\section{Diagnostic accuracy of standard MRI and DPP-TSO-Sag-FS- PDWI sequences compared to arthroscopy}

The number of MRI diagnostic results for the different grades of tears in each sequence was calculated, including TP, FN, FP, and TN (Supplementary material), which were compared to arthroscopic examination results. 
The diagnostic effectiveness of standard sequences and the new modified DPP-TSO-Sag-FS-PDWI sequence for the reader 1 was presented in Table 3, including sensitivity, specificity, accuracy, positive predictive values (PPV), and negative predictive values (NPV). Compared to standard sequences, the new modified DPP-TSO-Sag-FS-PDWI sequence showed superior sensitivity in the diagnosis of grade I (100\% vs. $75 \%$ ) and grade III injury ( $95.7 \%$ vs. $77.8 \%$ ), better specificity for both grade II ( $96.4 \%$ vs. $82.4 \%$ ), higher accuracy for grade III ( $92.9 \%$ vs. $78.6 \%)$ superior PPV for grade II (92.3\% vs. $53.9 \%$ ) and greater NPV for grade III ( $94.4 \%$ vs. $66.7 \%)$. Both sequences showed similar sensitivity for grade II injury $(85.7 \%$ vs. $87.5 \%)$, similar specificity for grade III ( $89.5 \%$ vs. $80 \%)$, similar accuracy for grade I (100\% vs. $97.6 \%)$ and grade II ( $92.9 \%$ vs. $83.3 \%)$. Furthermore, the specificity and PPV values of both sequences were the same for grade I injury (100\%). 
Table 3

The SE, SP, AC, PPV and NPV percentage values of three grades of ACL tears on standard MRI and the new modified DPP-TSO-Sag-FS-PDWI Sequences for reader 1 and reader 2

\begin{tabular}{|c|c|c|c|c|}
\hline Grade & & I & II & III \\
\hline \multirow[t]{4}{*}{ SE (\%) } & Standard sequences 1 & 75 & 87.5 & 77.8 \\
\hline & DPP-TSO-Sag-FS-PDWI 1 & 100 & 85.7 & 95.7 \\
\hline & Standard sequences 2 & 60 & 75 & 90.9 \\
\hline & DPP-TSO-Sag-FS-PDWI 2 & 100 & 84.6 & 91.7 \\
\hline \multirow[t]{4}{*}{ SP (\%) } & Standard sequences 1 & 100 & 82.4 & 80 \\
\hline & DPP-TSO-Sag-FS-PDWI 1 & 100 & 96.4 & 89.5 \\
\hline & Standard sequences 2 & 100 & 86.7 & 80 \\
\hline & DPP-TSO-Sag-FS-PDWI 2 & 100 & 93.1 & 88.9 \\
\hline \multirow[t]{4}{*}{ AC (\%) } & Standard sequences 1 & 97.6 & 83.3 & 78.6 \\
\hline & DPP-TSO-Sag-FS-PDWI 1 & 100 & 92.9 & 92.9 \\
\hline & Standard sequences 2 & 95.2 & 83.3 & 85.7 \\
\hline & DPP-TSO-Sag-FS-PDWI 2 & 100 & 90.5 & 90.5 \\
\hline \multirow[t]{4}{*}{ PPV (\%) } & Standard sequences 1 & 100 & 53.9 & 87.5 \\
\hline & DPP-TSO-Sag-FS-PDWI 1 & 100 & 92.3 & 91.7 \\
\hline & Standard sequences 2 & 100 & 69.2 & 83.3 \\
\hline & DPP-TSO-Sag-FS-PDWI 2 & 100 & 84.6 & 91.7 \\
\hline \multirow[t]{4}{*}{ NPV (\%) } & Standard sequences 1 & 97.4 & 96.6 & 66.7 \\
\hline & DPP-TSO-Sag-FS-PDWI 1 & 100 & 93.1 & 94.4 \\
\hline & Standard sequences 2 & 94.9 & 89.7 & 88.9 \\
\hline & DPP-TSO-Sag-FS-PDWI 2 & 100 & 93.1 & 88.9 \\
\hline
\end{tabular}

The diagnostic indicators of the reader 2 of standard sequences and the new modified DPP-TSO-Sag-FSPDWI sequence were presented in Table 3. Compared to standard sequences, the new modified DPP-TSOSag-FS-PDWI sequence showed greater sensitivity for grade I (100\% vs. $60 \%)$ and grade II injury $(84.6 \%$ vs. $75 \%)$, higher specificity for grade II ( $93.1 \%$ vs. $86.7 \%)$ and grade III ( $88.9 \%$ vs. $80 \%)$, better accuracy for all grades [(100\% vs. $95.2 \%)$, (90.5\% vs. $83.3 \%)$ and $(90.5 \%$ vs. $85.7 \%)]$ and superior PPV for grade II 
(84.6\% vs. $69.2 \%)$. Furthermore, both sequences showed similar sensitivity for grade III ( $91.7 \%$ vs. $90.9 \%)$, same specificity and PPV for grade I (100\%), and same NPV for grade III (88.9\%).

\section{Discussion}

The misdiagnosis rate of anterior cruciate ligament (ACL) tear of the knee joint is as high as $47 \%$ due to the confusion of high signal generated by synovial hyperplasia and partial volume effect on the standard MRI sequences [8]. A study revealed the evaluation of ACL tear by standard MRI sequences with additional oblique-sagittal plane showed high sensitivity and specificity $[18,15]$. Furthermore, another study using two additional oblique sequences (sagittal and coronal) to evaluate the ACL tear concluded that the use of oblique coronal and oblique sagittal MRI of the knee improved the diagnostic accuracy of the ACL tear [19]. Therefore, we performed this study to analyze the diagnostic accuracy by adding a new modified dual precision positioning of thin-slice oblique sagittal fat suppression proton-density weighted imaging (DPP-TSO-Sag-FS-PDWI) to standard MRI sequences. The diagnostic consistency of the new modified DPP-TSO-Sag-FS-PDWI sequence and arthroscopy was better than standard MRI sequences for both reader 1 ( $K=0.876$ vs. 0.620$)$ and reader $2(K=0.833$ vs. 0.683$)$ with good diagnostic repeatability ( $K=0.794$ vs. 0.598 ). Compared to standard sequences, the new modified DPP-TSO-Sag-FS-PDWI sequence can accurately classify and correctly diagnose the grades of ACL injury, whose sensitivity, specificity, accuracy, positive predictive value (PPV), and negative predictive value (NPV) were all greater than $84 \%$, especially for grade II injury (partial tear) as the PPV value was superior for the reader 1 (92.3\% vs. $53.9 \%)$ and reader $2(84.6 \%$ vs. $69.2 \%)$.

Previous studies have used standard MRI sequences with or without the additional sequence to diagnose an ACL tear. A previous study using 3.0-T MRI with standard sequences revealed that the specificity and accuracy of the partial injury $(97 \%, 95 \%)$ and complete injury $(99 \%, 97 \%)$, which are similar to present study. However, the sensitivity of the partial injury (77\%) was lower than our study (85.7\% or $84.6 \%)$ [20]. This indicates that although 3.0-T MRI is a highly accurate method for the diagnosis of ACL injuries, the new modified DPP-TSO-Sag-FS-PDWI sequence on a 1.5-T scanner with a single channel knee coil can achieve similar effects and further improve the diagnostic sensitivity to partial tears. There was a study of 149 patients with three different methods, including standard MRI sequences, patient knee in 17-degree flexion MRI technique, and additional sagittal-oblique sequence, and it revealed the sagittal-oblique sequence more sensitive $(p<0.001)$ than standard MRI sequences. The author mentioned the obliquesagittal technique can display the partial tear because of the double angulation which is following the course of the patient's ligament due to the approximate orientation of the external rotation of the foot [15]. The study using two additional oblique sequences (sagittal and coronal) to evaluate the ACL tear concluded that the usage of oblique coronal and oblique sagittal MRI of the knee improved the diagnostic accuracy of the ACL tear [19]. Furthermore, another study revealed the evaluation of ACL tear by standard MRI sequences with additional oblique-sagittal plane showed high sensitivity and specificity compared to a standard MRI protocol [18]. The results in these studies are similar to ours and we further found that our new modified DPP-TSO-Sag-FS-PDWI sequence can maximize the acquisition of ACL complete and true anatomical images and exclude the influence of anatomical differences between individuals, which is 
helpful to improve the authenticity and accuracy of ACL scanning images and to improve the diagnostic accuracy of ACL injury with its three grades.

To our knowledge, this is the first study to display and diagnose the ACL injury with its three grades by using a dual precision positioning based on axial and coronal localization. Our study not only validated the diagnostic accuracy of standard sequences used to diagnose ACL injury and its grades but also added a new modified dual precision positioning of thin-slice oblique sagittal sequence to improve the diagnostic accuracy. Therefore, the unnecessary arthroscopic surgery can be prevented and the financial burden can be decreased [9]. Additionally, our scanning method is simple, the scanning time is moderate, and there is no special requirement for the MR scanner and the patient's position. Therefore, it is very suitable for popularization and application in clinical work.

The present study has several limitations. Firstly, the sample size was small which may be caused bias. Secondly, the new modified DPP-TSO-Sag-FS-PDWI sequence cannot identify meniscus injury simultaneously, which is caused by a narrow scanning range. Lastly, our MR scanner and knee coils were relatively backward, and given the time required to scan and patient tolerance, our sequence was compared only with standard sequences, not with 3D isotropic or other sequences. In the future, we will also report the related study by using this sequence to distinguish the anteromedial bundle and posterolateral bundle of the ACL.

\section{Conclusions}

The new modified DPP-TSO-Sag-FS-PDWI sequence can clearly display the ligament injury on one or continuous levels by maximizing the acquisition of ACL complete and true anatomical images and excluding the influence of anatomical differences between individuals. It can improve the diagnostic accuracy with good diagnostic repeatability and classify three different grades of ACL injury accurately, especially for grade II injury. This new modified sequence also assists the physicians to reduce unnecessary arthroscopic intervention and provides a precise diagnostic and treatment basis for ACL injury.

\section{References}

1. Irarrázaval, S., Albers, M., Chao, T. \& Fu, F. H. Gross, Arthroscopic, and Radiographic Anatomies of the Anterior Cruciate Ligament: Foundations for Anterior Cruciate Ligament Surgery. Clinics in sports medicine, 36 (1), 9-23 https://doi.org/doi:10.1016/j.csm.2016.08.002 (2017).

2. Acevedo, R. J., Rivera-Vega, A., Miranda, G. \& Micheo, W. Anterior cruciate ligament injury: identification of risk factors and prevention strategies. Current sports medicine reports, 13 (3), 186191 https://doi.org/doi:10.1249/jsr.0000000000000053 (2014).

3. Siegel, L., Vandenakker-Albanese, C. \& Siegel, D. Anterior cruciate ligament injuries: anatomy, physiology, biomechanics, and management. Clinical journal of sport medicine: official journal of the 
Canadian Academy of Sport Medicine, 22 (4), 349-355

https://doi.org/doi:10.1097/JSM.0b013e3182580cd0 (2012).

4. Boden, B. P., Sheehan, F. T., Torg, J. S. \& Hewett, T. E. Noncontact anterior cruciate ligament injuries: mechanisms and risk factors. The Journal of the American Academy of Orthopaedic Surgeons, 18 (9), 520-527 https://doi.org/doi:10.5435/00124635-201009000-00003 (2010).

5. Zantop, T., Petersen, W. \& Fu, F. H. Anatomy of the anterior cruciate ligament. Operative Techniques in Orthopaedics, 15 (1), 20-28 https://doi.org/doi:10.1053/j.oto.2004.11.011 (2005).

6. Rossbach, B. P. et al. Indications requiring preoperative magnetic resonance imaging before knee arthroscopy. Archives of medical science: AMS, 10 (6), 1147-1152

https://doi.org/doi:10.5114/aoms.2014.47825 (2014).

7. Syal, A. \& Chudasama, C. H. Clinical examination, magnetic resonance imaging and arthroscopic correlations of ligament and menisci injuries of knee joint. Journal of Arthroscopy and Joint Surgery, 2 (1), 3-8 https://doi.org/doi:10.1016/j.jajs.2014.12.003 (2015).

8. Li, K. et al. The diagnostic accuracy of magnetic resonance imaging for anterior cruciate ligament injury in comparison to arthroscopy: a meta-analysis. Scientific reports, 7 (1), 7583 https://doi.org/doi:10.1038/s41598-017-08133-4 (2017).

9. Orlando Junior, N., de Souza Leao, M. G. \& de Oliveira, N. H. Diagnosis of knee injuries: comparison of the physical examination and magnetic resonance imaging with the findings from arthroscopy. Revista brasileira de ortopedia, 50 (6), 712-719 https://doi.org/doi:10.1016/j.rboe.2015.10.007 (2015).

10. Vassalou, E. E., Klontzas, M. E., Kouvidis, G. K., Matalliotaki, P. I. \& Karantanas, A. H. Rotational Knee Laxity in Anterior Cruciate Ligament Deficiency: An Additional Secondary Sign on MRI. AJR American journal of roentgenology, 206 (1), 151-154 https://doi.org/doi:10.2214/AJR.15.14816 (2016).

11. Chagas-Neto, F. A. et al. Diagnostic performance of 3D TSE MRI versus 2D TSE MRI of the knee at $1.5 \mathrm{~T}$, with prompt arthroscopic correlation, in the detection of meniscal and cruciate ligament tears. Radiologia brasileira, 49 (2), 69-74 https://doi.org/doi:10.1590/0100-3984.2015.0042 (2016).

12. Lee, J. E. et al. Evaluation of Selective Bundle Injury to the Anterior Cruciate Ligament: T2-Weighted Fast Spin-Echo 3-T MRI With Reformatted 3D Oblique Isotropic (VISTA) Versus 2D Technique. AJR American journal of roentgenology, 209 (5), W308-W316 https://doi.org/doi:10.2214/AJR.16.17659 (2017).

13. Colombet, P., Dejour, D., Panisset, J. C., Siebold, R. \& French Arthroscopy, S. (2010) Current concept of partial anterior cruciate ligament ruptures. Orthopaedics \& traumatology, surgery \& research: OTSR 96 (8 Suppl):S109-118. doi:10.1016/j.otsr.2010.09.003

14. Mohankumar, R. \& Naraghi, A. M. (2018) Magnetic Resonance Imaging for the Diagnosis and Treatment of Anterior Cruciate Ligament Tears.51-60.e51. doi:10.1016/b978-0-323-38962-4.00010-2

15. Nenezic, D. \& Kocijancic, I. The value of the sagittal-oblique MRI technique for injuries of the anterior cruciate ligament in the knee. Radiology and oncology, 47 (1), 19-25 https://doi.org/doi:10.2478/raon-2013-0006 (2013). 
16. Hong, S. H. et al. Grading of anterior cruciate ligament injury. Diagnostic efficacy of oblique coronal magnetic resonance imaging of the knee. Journal of computer assisted tomography, 27 (5), 814819 https://doi.org/doi:10.1097/00004728-200309000-00022 (2003).

17. Sim, J. \& Wright, C. C. The kappa statistic in reliability studies: use, interpretation, and sample size requirements. Physical therapy, 85 (3), 257-268 (2005).

18. Ghasem Hanafi, M., Momen Gharibvand, M., Jaffari Gharibvand, R. \& Sadoni, H. Diagnostic Value of Oblique Coronal and Oblique Sagittal Magnetic Resonance Imaging (MRI) in Diagnosis of Anterior Cruciate Ligament (ACL) Tears. Journal of medicine and life, 11 (4), 281-285 https://doi.org/doi:10.25122/jml-2018-0015 (2018).

19. Kosaka, M. et al. Oblique coronal and oblique sagittal MRI for diagnosis of anterior cruciate ligament tears and evaluation of anterior cruciate ligament remnant tissue., 21 (1), 54-57 https://doi.org/doi:10.1016/j.knee.2013.04.016 (2014).

20. Van Dyck, P. et al. Three tesla magnetic resonance imaging of the anterior cruciate ligament of the knee: can we differentiate complete from partial tears? Skeletal radiology, 40 (6), 701-707 https://doi.org/doi:10.1007/s00256-010-1044-8 (2011).

\section{Figures}

\section{Figure 1}

Scanning method for the new modified DPP-TSO-SAG-FS-PDWI sequence. The left knee of a 26-year-old man was displayed on $a, b$ and c. ACL on the new modified DPP-TSO-Sag-FS-PDWI sequence (c) were performed along its anatomic direction on axial and coronal PDWI (as yellow line marked on a and $b$ ). Its complete structure was shown in the yellow range (arrow on c) with low signal intensity.

\section{Figure 2}

Normal ACL presence on MRI and arthroscopy of a 14-years-old female teenager. The ACL was shown on sagittal T1WI (a), coronal T2WI (b), Sagittal PDWI (c), coronal PDWI (d), and axial PDWI (e). It was tilted from the tibial to the condyle and the attachment was inserted from the condyle to the notch with low signal intensity (arrows). The ligament could be displayed completely on one level of the new modified DPP-TSO-Sag-FS-PDWI (arrow on f). The normal appearance of ACL was proved on arthroscopy (arrow on g).

\section{Figure 3}


Grade I injury of ACL presence on MRI and arthroscopy of a 34-years-old woman. The shape and signal intensity of the ACL (arrows) was similar to the normal ligament on sagittal T1WI (a), coronal T2WI (b), Sagittal PDWI (c), coronal PDWI (d), and axial PDWI (e) with effusion of knee joint on PDWI. The ligament was tortuous as "S-shaped" and localized edema with increased signal intensity in the tibial part on the new modified DPP-TSO-Sag-FS-PDWI (arrow on f). Arthroscopy showed the ligament was loose with a hematoma in the anterior tibial part of the ligament (arrow on g).

\section{Figure 4}

Grade II injury presence on MRI and arthroscopy of a 35-years-old man. The remaining structure of ACL was shown on sagittal and coronal T1WI (arrows on a and b). The ligament lost continuity and turned thinner, and its shape was distorted with a high signal on sagittal PDWI (arrow on c) and coronal PDWI (arrow on d). The signal of ACL increased and surrounded by the fluid on axial PDWI (arrow on e). It could be shown on the new modified DPP-TSO-Sag-FS-PDWI (f) that most of the ACL was swollen with the increased signal. A tear was seen at the posterior femoral part of the ACL, surrounded by fluid, and the free edge was reflexed (arrow), which was corresponded to the arthroscopic image. Arthroscopy showed that most remaining part of the ACL was still attached to the origin site and a small portion of the fibrous was tearing down in the middle of ACL (arrow on g).

\section{Figure 5}

Grade III injury presence on MRI and arthroscopy of a 23-years-old woman. The absence of ligament integrity and continuity were shown on sagittal, and coronal T1WI (arrows on a and b). Remaining filamentous ACL with surrounding liquid (arrows) still could be seen on sagittal PDWI (c), coronal PDWI (d), and axial PDWI (e), which would cause misdiagnosis. The ligament was completely separated into two parts and the two damaged ends with their intermediate filled fluids (arrow) were displayed on the new modified DPP-TSO-Sag-FS-PDWI ( $f$ ). Arthroscopy found the ligament was divided into two parts without continuity and completely separated, one of the damaged edges faced up (arrow on g).

\section{Supplementary Files}

This is a list of supplementary files associated with this preprint. Click to download.

- DistributionofTPFNFPandTN.docx

- Flowchart.jpg 